\title{
Impact of Blood Culture Utilization on Antibiotics Choice in Chemotherapy Induced Febrile Neutropenia in Solid Tumours
}

\author{
S Gilani*, G Hegarty, J Whithurst and Z Rehman \\ University Hospital of North Midlands, United Kingdom \\ *Corresponding Author: S Gilani, University Hospital of North Midlands, United \\ Kingdom.
}

Received: April 12, 2021

Published: May 11, 2021

(C) All rights are reserved by $\mathbf{S}$ Gilani., et al.

\begin{abstract}
Blood cultures are important tools for the evaluation of sepsis in febrile neutropenia (FN) patients. Febrile neutropenia remains a serious complication of anticancer systemic chemotherapy with increased mortality worldwide. NICE (National Institute of Clinical Excellence) UK issued clinical practice guideline to prevent and manage febrile neutropenia in cancer patients [1]. There was also a genuine concern about diagnostic dilemma and management of febrile neutropenia during recent pandemic of severe acute respiratory syndrome coronavirus 2 (SARS-CoV-2) associated disease COVID-19 [2]. Healthcare resources were already limited and were overwhelmed rapidly by large influx of patients [3]. Routine medical care including management of cancer patient was severely disrupted [4]. This led to the developments of rapid guidelines to manage cancer patients across the world [5]. NICE and other bodies issued rapid guidelines to manage febrile neutropenia during COVID-19 period [6]. Majority of guidelines consider blood culture an essential part in assessment of sepsis in febrile neutropenia [1]. This prospective study was done in hospitalized patients who developed febrile neutropenia while on chemotherapy, looking at various factors and their impact on outcome. The objective of this study was to evaluate whether antibiotic choice is affected by blood culture utilization in febrile neutropenia [7].
\end{abstract}

Keywords: SARS-CoV-2; COVID-19; Febrile Neutropenia

\section{Aims}

In this study relationship of various factors and their impact on short term outcome on hospitalized patients with chemotherapy induced febrile neutropenia was examined including blood cultures and frequency of positive bacterial cultures from blood stream on antimicrobial decision-making.

\section{Material and Methods}

A prospective observational study was conducted for a year on cohort of adult patients with solid tumors receiving chemotherapy that developed febrile neutropenia in cancer center at UHNM in UK. Hospital databases were used to examine various factors and data was statistically analyzed using univariate and multivariate approaches.

\section{Results and Discussion}

There was total 37 events identified in 35patients over a period of one year that developed chemotherapy associated febrile neutropenia. Mean age was $57(S D=13)$ years. All of them received chemotherapy within four weeks. Three quarters were females and rest was males. Prophylactic antibiotics were given to $13 \%$ (5/37) of patients and prophylactic Granulocyte colony-stimulating factor (G-CSF or GCSF) was given to $21 \%$ (8/37). This had no impact on the outcome of patients. Average MASCC Score was 22 (MASCC=Multinational Association for Supportive Care in Cancer score) [8].

The mean length of hospital stay (LOS) was 4.6 days ( $\mathrm{SD}=2.6$ ). The length of hospital stay was not related to cancer type, age, MAS- 
CC score, or prophylactic use of antibiotics or GCSF. But it showed direct correlation with peak C-reactive protein (CRP) and delay in starting antibiotics $(\mathrm{P}=0.0189)$. The mean delay in starting antibiotics was 1 hour and 40 minutes. The mean number of days of intravenous antibiotics use was 3.4 (SD = 1.5). Almost 67\% $(25 / 37)$ of the patients received intravenous antibiotics for more than 48 hours. Antibiotics were changed to oral route subsequently. Therefore, total mean number of days of antibiotics were 6.7 days (SD $=2.7$ ). There were about 5\% (2/37) patients with central venous catheter. Positive blood culture yield was10\% (4/37) in detecting bacteremia from the blood samples taken for blood cultures. This did not lead to change in antibiotics in either. In this study $97 \%$ of patients received guideline based antibiotics with 95\% positive outcome.
Majority of the antibiotics were combination of intravenous piperacillin with tazobactam followed by oral co-amoxiclav or clarithromycin in case of penicillin allergy. In 3\% of the patients, vancomycin was added empirically due to suspected central venous catheter (CVC) related infection. Overall, short term outcome was encouraging with 95\% (35/37) of patients discharged home with resolution of FN. Two patients become unwell and were moved to intensive care unit.

Finding of this study are mostly in line with national [9] and international data [10]. This study heighted a limited impact of blood culture utilization on detection of bacteremia and choice of antibiotics.

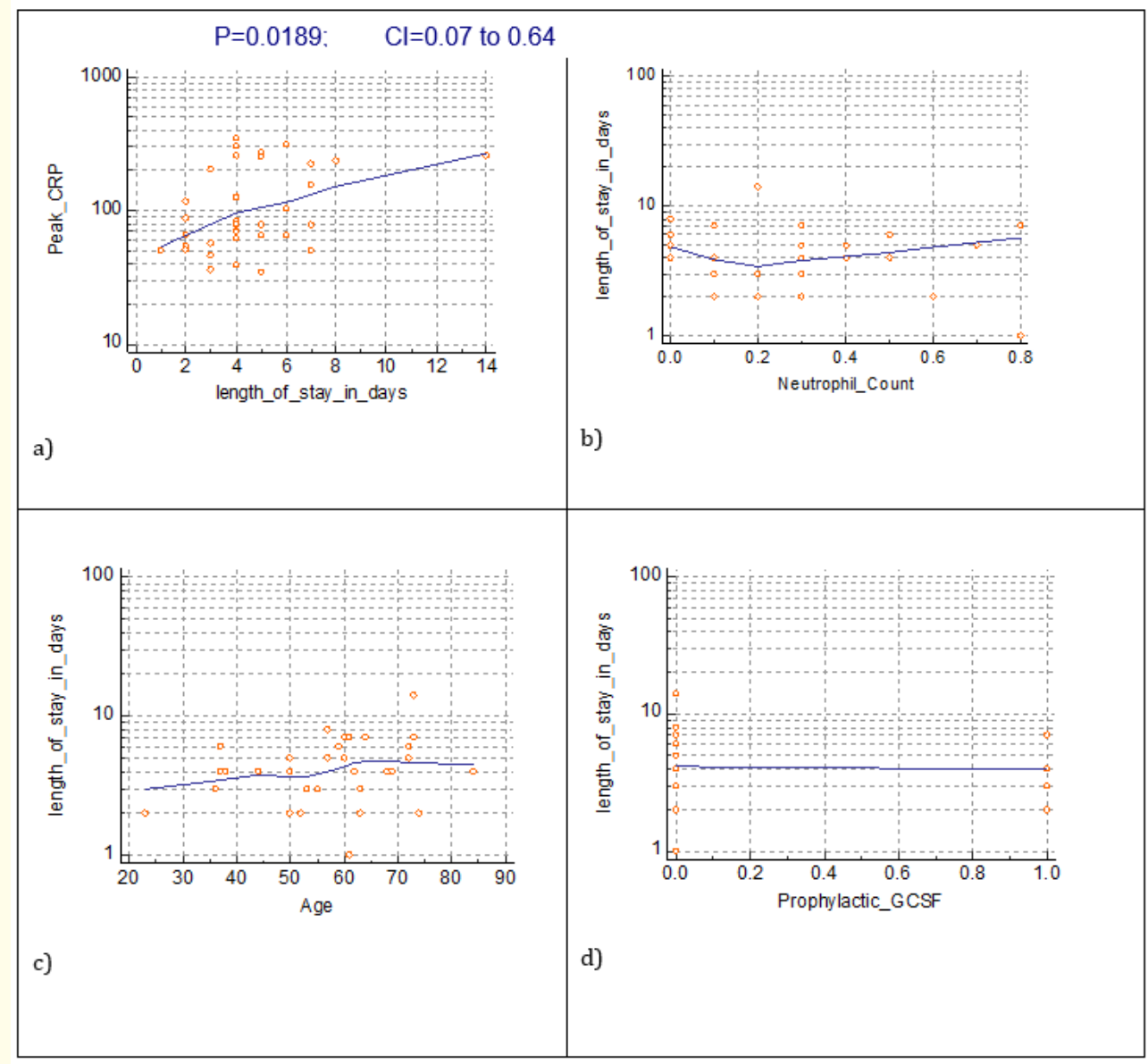

Figure 1: Correlation between Length of Stay in hospital (LOS) and age, CRP, Neutrophil count and prophylactic GCSF). 


\section{Conclusion}

Delay in starting antibiotics was directly related to the length of stay in hospital. This study highlighted limited impact of blood culture utilization on physician's choice of antibiotics. Although use of blood cultures as a diagnostic tool has increased over the years along with improvements in techniques and results but majority of blood cultures come back as negative, and some are contaminated. In patients admitted with neutropenic sepsis, blood cultures may have a limited role in the diagnosis of bacterial infection. Decisions of choice and review of antimicrobial prescribing should combine clinical assessment with a range of diagnostic information which includes, but is not limited to, culture-based microbiology. In this study using real-world data from our cancer center in England over a year, parenteral antimicrobials were prescribed to majority of patients admitted with neutropenic sepsis. But a micro-organism was identified in just $10 \%$ of the patients who had a blood culture. Therefore, blood culture had little impact on physician's choice of antibiotics. Therefore, newer rapid bacterial diagnostics, particularly blood culture-independent tests, are urgently needed for hospitalized febrile neutropenia patients to improve pathogen detection and better antibiotic utilization especially in low-income countries. This is especially true in the current climate of COVID19 pandemic.

\section{Acknowledgement}

No potential competing interest was reported by the authors.

\section{Bibliography}

1. NICE - Neutropenic sepsis: prevention and management of neutropenic sepsis in cancer patients. Clinical guidelines, CG151 (2012).

2. Remuzzi A and Remuzzi G. "COVID-19 and Italy: what next?" Lancet 395.10231 (2020): 1225-1228.

3. Hollander JE and Carr BG. "Virtually perfect? Telemedicine for covid-19". The New England Journal of Medicine 382.18 (2020): 1679-1681.

4. Ueda M., et al. "Managing cancer care during COVID-19 pandemic: agility and collaboration toward a common goal". Journal of the National Comprehensive Cancer Network 18.4 (2020).

5. Giuseppe Curigliano., et al. "Recommendations for triage, prioritization and treatment of breast cancer patients during the COVID-19 pandemic". Breast 52 (2020): 8-16.
6. NICE Rapid Guidelines accessed on 8th August (2020).

7. Aaron M Harris., et al. "Influence of Antibiotics on the Detection of Bacteria by Culture-Based and Culture-Independent Diagnostic Tests in Patients Hospitalized With CommunityAcquired Pneumonia". Open Forum Infectious Diseases 4.1 (2017): ofx014.

8. Jean Klastersky., et al. "The Multinational Association for Supportive Care in Cancer Risk Index: A Multinational Scoring System for Identifying Low-Risk Febrile Neutropenic Cancer Patients". Journal of Clinical Oncology 18.16 (2000): 30383051.

9. Jason D Wright., et al. "Deviations From Guideline-Based Therapy for Febrile Neutropenia in Cancer Patients and Their Effect on Outcomes". JAMA Internal Medicine 173.7 (2013): 559-568.

10. Mabel Wong., et al. "Prospective Audit of Post-chemotherapy Febrile Neutropenia in Patients with Solid Cancer and Lymphoma in Two Singaporean Cancer Centres". Journal of Annals Academy of Medicine 41.7 (2012): 288.

\section{Volume 5 Issue 6 June 2021}

(c) All rights are reserved by S Gilani., et al. 